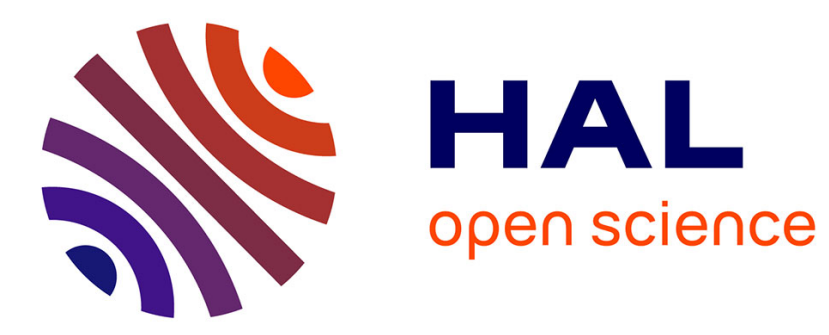

\title{
Planar Path Following of 3-D Steering Scaled-Up Helical Microswimmers.
}

Tiantian Xu, Gilgueng Hwang, Nicolas Andreff, Stéphane Régnier

\section{To cite this version:}

Tiantian Xu, Gilgueng Hwang, Nicolas Andreff, Stéphane Régnier. Planar Path Following of 3-D Steering Scaled-Up Helical Microswimmers.. IEEE Transactions on Robotics, 2015, 31 (1), pp.117127. 10.1109/TRO.2014.2380591 . hal-01303497

\section{HAL Id: hal-01303497 https://hal.science/hal-01303497}

Submitted on 28 Apr 2016

HAL is a multi-disciplinary open access archive for the deposit and dissemination of scientific research documents, whether they are published or not. The documents may come from teaching and research institutions in France or abroad, or from public or private research centers.
L'archive ouverte pluridisciplinaire HAL, est destinée au dépôt et à la diffusion de documents scientifiques de niveau recherche, publiés ou non, émanant des établissements d'enseignement et de recherche français ou étrangers, des laboratoires publics ou privés. 


\title{
Planar Path Following of 3D Steering Scaled-up Helical Microswimmers
}

\author{
Tiantian Xu, Gilgueng Hwang, Nicolas Andreff and Stéphane Régnier
}

\begin{abstract}
Helical microswimmers capable of propulsion at low Reynolds numbers have great potential for numerous applications. Several kinds of artificial magnetic actuated helical microswimmers have been designed by researchers. However, they are primarily open-loop controlled. This paper aims to investigate methods of closed-loop control of a magnetic actuated helical swimmer at low Reynolds number by using visual feedback. For many in-vitro applications, helical swimmers should pass through a defined path, for example along channels, without any prerequisite on the velocity profile along the path. Therefore, the main objective of this paper is to achieve a velocity-independent planar path following task. Since the planar path following is based on 3D steering control of the helical swimmer, a 3D pose estimation of a helical swimmer is introduced based on the realtime visual tracking with a stereo vision system. The contribution of this paper is in two parts: the 3D steering of a helical swimmer is demonstrated by visual servo control; and the path following of a straight line with visual servo control is achieved, then compared to open-loop control. We further expect that with this visual servo control method, the helical swimmers will be able to follow reference paths at the microscale.
\end{abstract}

Index Terms-helical swimmer, magnetic actuation, 3D steering, path following, visual servoing, 3D pose estimation.

\section{INTRODUCTION}

Helical microswimmers capable of propulsion at low Reynolds numbers have been proposed for numerous applications, ranging from in vitro tasks on labs-on-chips (e.g. transporting and sorting micro objects; mechanical components micro assembly [1]...) to in vivo applications for minimally invasive medicine (e.g. targeted drug delivery; brachytherapy; hyperthermia [2]...), due to their micro sizes and accessibility to tiny and clustered environments. However, at the microscale, the fluid becomes extremely viscous and the Reynolds number of the microswimmers decreases dramatically. The corkscrew type rotating propulsion used by some natural micro-organism, for example E. coli bacteria, is one of the swimming technique suitable for swimming at low Reynolds numbers [3], [4]. Artificial helical microswimmers, driven by a rotating magnetic field, are inspired by E. coli bacteria [5]. They are able to convert rotary motion to a linear motion.

T. XU is with ISIR - Institut des Systèmes Intelligents et Robotique, UPMC, Paris, France \& Department of Mechanical and Automation Engineering, the Chinese University of Hong Kong, Hong Kong, China. tiantian.xueisir.upme.fr, b133656emailserv. cuhk. edu.hk

G. Hwang is with Laboratoire de Photonique et de Nanostructures, Marcoussis, France. gilgueng.hwang@lpn.cnrs.fr

$\mathrm{N}$. Andreff is with FEMTO-ST Institute, UFC/CNRS/ENSMM/UTBM, Besançon, France. nicolas.andreffefemto-st. fr

S. Régnier is with ISIR - Institut des Systèmes Intelligents et Robotique, UPMC, Paris, France. stephane.regniereupmc.fr
For more than ten years, researchers have developed several kinds of magnetic actuated helical swimmers or crawlers from several millimeters [6], [7], [8] down to a few micrometers [9], [10], [11], [12]. Ghosh et al. proved that helical swimmers with an overall length of $2 \mu \mathrm{m}$ could follow a curved trajectory, for example "R@H", under the action of a preprogrammed magnetic field [13]. Mahoney et al. showed an open-loop controlled helical swimmer with an overall length of $5 \mathrm{~mm}$, which enabled a U-turn trajectory in the vertical plane [14]. Tottori et al. fabricated helical swimmers ranging from $4 \mu \mathrm{m}$ to $64.5 \mu \mathrm{m}$, which enabled 3D motion and could carry a sphere as well as raise it up [15]. As far as we know, the existing helical swimmers are primarily open-loop controlled, mainly due to the challenges of visual feedback and tracking through micro and nanoscopic imaging.

A closed-loop control of helical swimmers is necessary for precise motion in presence of perturbations generated by thermal noise [16] or drifting due to boundary effects [17]. The aim of this paper is to control a magnetic actuated helical swimmer using visual feedback. Visual servo controls show good results with industrial robots [18], [19] or unmanned vehicles [20], but have not been applied to helical swimmers. A Scaled-up Helical Microswimmer (SHM) at the millimeter scale will be used, in order to eliminate the optical tracking problems. The fluid motion around the helical swimmer is only characterized by the Reynolds number. Therefore, the swimming properties of helical swimmers should be the same if they swim at the same Reynolds numbers, which makes a dimensionless study possible [8]. At a larger scale, to emulate the environment of microscale swimmers swimming at low Reynolds numbers, one can use a more viscous liquid, such as glycerol. The SHMs facilitate fabrication and can be observed with regular optical devices. They are therefore good candidates for preliminary visual servoing experiments. Once the visual servo control is validated on the SHMs, it can be applied to helical microswimmers by using suitable optical devices with a higher resolution in future works. The SHMs can be scaled down to the microscale with the recently developed microswimmer micro-fabrication processes, such as the self-scrolling of a bilayer [9], the glancing angle deposition method [13], and microscale 3D lithography [15].

For most in-vitro applications of helical swimmers, such as labs-on-chips, they should pass through a defined path, for example pass through micro channels, with a constant altitude without any prerequisite on the velocity profile along the path. Therefore, the main objective of this paper is to establish a velocity-independent control law of the helical system to follow a planar reference path based on 3D steering. The 
steering is in the 3D space, because the helical swimmer has an inclination angle in order to decrease the friction with the substrate. In this paper, Section II depicts the visual servo control architecture of the path following based on its 3D steering. Section III presents the modelling of the magnetic actuated helical swimmer, including the linear propulsion and the 3D steering. Section IV details the kinematic equations of the planar path following task of a helical swimmer, and the control law design. Section V presents the visual servo control for 3D steering based on the orientation error of the helical swimmer. Section VI presents the overview of the experimental system, including the magnetic actuation system, stereo vision system, and the SHM at low Reynolds number. Section VII introduces a 3D pose estimation including the position and the orientation of the SHM based on its real-time tracked barycenter and axis. The results of the 3D steering and the planar path following are given in Section VIII. Finally, Section IX concludes the paper.

\section{CONTROL ARCHITECTURE}

A planar reference path, as shown in Fig. 1, is defined to be followed by a helical swimmer. A helical swimmer can be considered as a unicycle-like nonholonomic system, because one helical swimmer cannot generate a lateral motion voluntarily. As explained in [21], "Nonholonomic systems are, roughly speaking, mechanical systems with constraints on their velocity that are not derivable from position constraints". All the lateral motions applied on a helical swimmer generated by thermal noise [16], or drifting due to boundary effect [17] can be considered as a perturbation, and should be corrected. Compensation of thermal noises is both a motivation of the closedloop control and a major technical challenge. Concerning the former, it is indeed very delicate to follow a time-dependent trajectory (e.g. to follow a virtual robot) in presence of thermal noise because thermal noise will yield time delays with the virtual robot on the trajectory and thus, the geometrical path will not be achieved. As for the latter, we have not investigated yet where the frontier lies between efficient propulsion at low Reynolds number and predominance of thermal noise.

Moreover, the velocity amplitude of a helical swimmer can not be reliably achieved in many cases. The first reason for that is the well-known cut-off frequency of the velocity amplitude [6], which imposes a strong saturation of the available amplitude. As we have shown in [8], this cut-off frequency depends on many uncontrolled factors (microfabrication quality and environmental viscosity, for the main ones). The second reason is related to the potential stiction of the helix onto the substrate, which creates a distortion in velocity amplitude. The third reason is related to the viscosity at low scales (through the Reynolds number). Indeed, at low scales, we are not sure that the fluid is really homogeneous (inherently or due to the presence of micro or nano-dirt in a non totally clean room). This is especially true when dealing with biological fluids (blood, cell culture) where the medium is definitely heterogeneous or, at least, where the cells represent mobile obstacles that need to be pushed aside to allow for the helical swimmer to follow the expected trajectory.
Disturbances have several origins (thermal noise, friction, nonhomogeneous fluid, cut-off frequency, whatever). These disturbances split in two effects. The first one is a lateral deviation with respect to the desired path and the second one is a delay with respect to the time-varying reference on the trajectory. Namely, the helical swimmer can effectively be on the desired trajectory but it is late. In such a case, trajectory tracking algorithms tend to resume the delay by "cutting the curves" (Fig. 1), whereas path planning algorithms consider that there is no error since the path is time-independent. Note that both kinds of algorithms tend to reject the lateral deviation. Our proposition is thus to merge non-holonomous path following algorithms with the specificity of the helical swimmer actuation. To do so, we chose to decompose the problem in two tasks: the first one is to keep a constant altitude and the other is to follow a path in the horizontal plane.

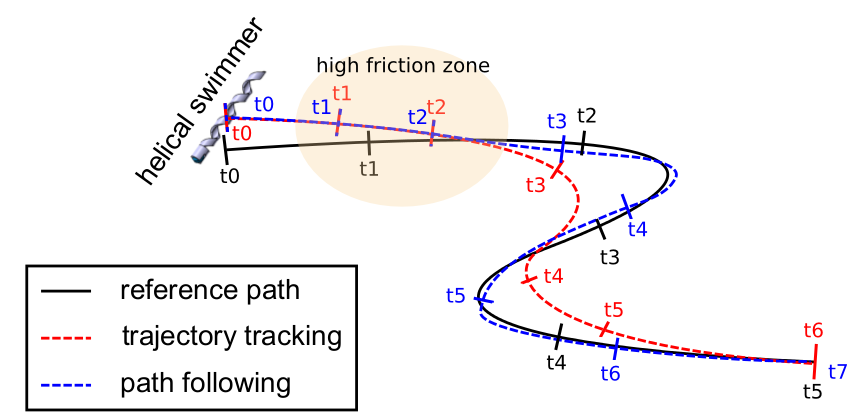

Fig. 1: Comparison between path following and trajectory tracking algorithms in presence of strong disturbances on the helical swimmer velocity (e.g. friction on substrate, nonhomogeneous fluid).

The planar path following of a helical swimmer is based on its steering similar to a unicycle, as presented by a blockdiagram in Fig. 2. The notation will be detailed in Section IV and VII. The steering is in the 3D space, because the helical swimmer has an inclination angle to maintain its altitude as well as to decrease friction with the substrate. The realtime position and orientation of the helical swimmer can be obtained by a 3D pose estimation method based on two images taken by a stereo vision system, which will be introduced in Section VII. The distance and orientation error between the helical swimmer and the reference path can be then obtained and used to deduce the target orientation of the helical swimmer, which will be detailed in Section IV. The control of the magnetic field for the 3D steering based on the orientation error will be detailed in Section V.

\section{Modelling of MAGnetic ACtUATED Helical SWIMMERS}

\section{A. Magnetic force and torque}

All magnetized objects are subjected to both force and torque within an externally imposed magnetic field. The magnetic force and torque induced on a magnetized object are expressed as follows [22]:

$$
\mathbf{f}_{m}=\int_{V_{m}}(\mathbf{M} \cdot \nabla) \mathbf{B} \mathrm{d} V_{m}
$$




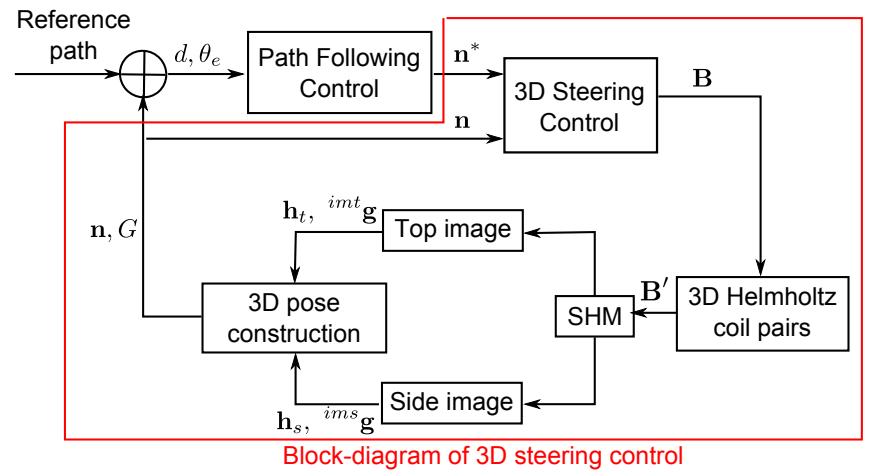

Fig. 2: Block-diagram of the visual servo control for planar path following based on 3D steering of helical swimmers.

$$
\tau_{m}=\int_{V_{m}} \mathbf{M} \times \mathbf{B} \mathrm{d} V_{m}
$$

where $V_{m}$ is the volume of the magnetized object, $\mathbf{B}$ is the flux density of the applied field ( $\mathrm{T}), \mathbf{M}$ is the magnetization of the object $(\mathrm{A} / \mathrm{m})$.

In this paper, the helical swimmer is actuated by a rotating magnetic field generated by a 3D Helmholtz coils system. The magnetic field can be considered as uniform. Therefore, the helical swimmer is driven by magnetic torque only.

\section{B. Inclination angle and direction angle}

In order to describe more clearly the movement of the helical swimmer, we define herein two angles: the inclination angle $\theta_{i}$ and the direction angle $\theta_{d}$ as shown in Fig. 3 .

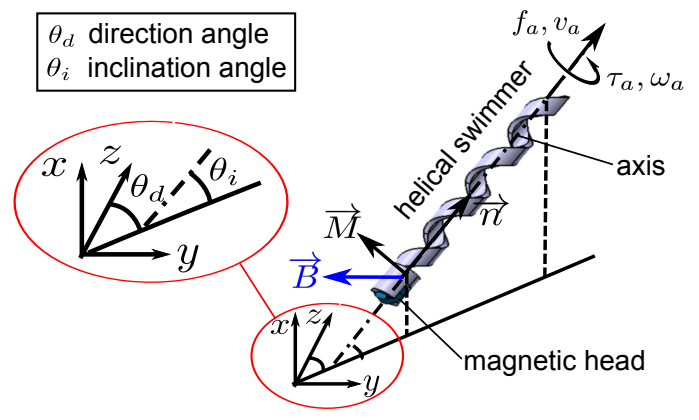

Fig. 3: Magnetic helical swimmer steering model. The inclination angle and direction angle of steering are respectively $\theta_{i}$ and $\theta_{d}$.

The inclination angle $\theta_{i}$ is defined as the angle between the axis of the helical swimmer and the horizontal plane $y O z$. This inclination angle is very important to control the height of the helical swimmer. The propulsive force projected on the upward direction can be increased by increasing the inclination angle or rotation frequency. If the propulsive force created by the helical swimmer projected on the upward direction is higher than its own gravity, the helical swimmer will go upwards. Otherwise, the helical swimmer will go downwards. Helical swimmers are kept inclined in order to decrease the surface friction as well.
The direction angle $\theta_{d}$ is defined as the angle between the $z$ axis and the projection of the axis of the helical swimmer on the horizontal plane. By controlling the direction angle of the helical swimmer, its forward direction projected on the horizontal plane can be changed.

The normal vector of the helical swimmer axis is noted as $\mathbf{n}$, which is defined as the orientation of the SHM, and can be expressed as:

$$
\mathbf{n}=\left[\begin{array}{c}
n_{x} \\
n_{y} \\
n_{z}
\end{array}\right]=\left[\begin{array}{c}
\sin \theta_{i} \\
\cos \theta_{i} \sin \theta_{d} \\
\cos \theta_{i} \cos \theta_{d}
\end{array}\right]
$$

\section{Modelling of linear advance}

Purcell proved in his paper that the nonfluidic applied force $\left(f_{a}\right)$ and nonfluidic applied torque $\left(\tau_{a}\right)$ on a helical swimmer along its axis are linearly related to its propulsion velocity $\left(v_{a}\right)$ and angular speed $\left(\omega_{a}\right)$ along its axis with four principal quantities described by a symmetric propulsion matrix [3]:

$$
\left[\begin{array}{l}
f_{a} \\
\tau_{a}
\end{array}\right]=\left[\begin{array}{ll}
a & b \\
b & c
\end{array}\right]\left[\begin{array}{l}
v_{a} \\
\omega_{a}
\end{array}\right]
$$

The parameters $\mathrm{a}, \mathrm{b}$, and $\mathrm{c}$ encapsulate the geometric and environmental properties of the helical swimmer. The nonfluidic applied forces are gravity, buoyancy, friction and magnetic forces, while the only nonfluidic torque is the magnetic torque.

The advance velocity $v_{h}$ is defined as the linear velocity projected on the horizontal plane, which can be expressed as:

$$
v_{h}=v_{a} \cos \theta_{i}
$$

where $v_{a}$ is the propulsion velocity along its axis, $\theta_{i}$ is the inclination angle.

\section{Planar Path Following}

\section{A. Notations}

The notations of the planar path following of the helical swimmer are depicted in Fig. 4:

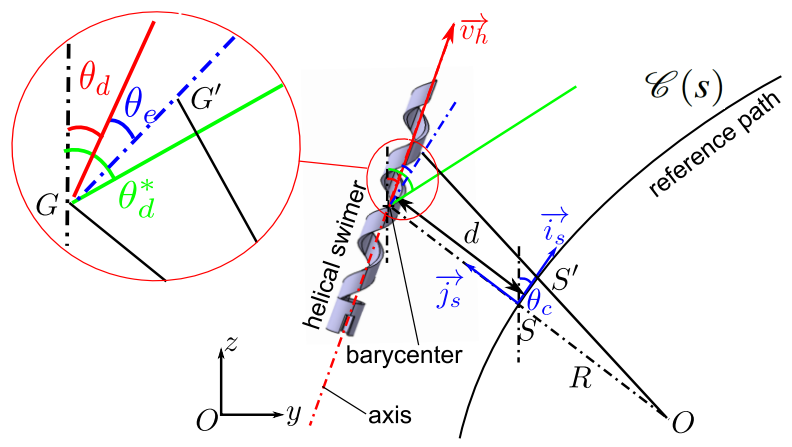

Fig. 4: Helical swimmer modelling of the planar path following.

- $\mathscr{C}(s)$ is the predefined reference path with respect to the curvilinear coordinate $s$ of the curve. The curve should be twice differentiable in $\mathbb{R}^{2}$ [23], [24].

- $\theta_{d}$ is the real-time direction angle of the SHM. 
- $v_{h}$ is the advance velocity of the SHM on the horizontal plane.

- $G$ and $G^{\prime}$ are the positions of the SHM's barycentre at time $t$ and $t+\mathrm{d} t$.

- $s$ is the curvilinear coordinate of $\mathscr{C}(s)$. $S$ and $S^{\prime}$ are the nearest points on the path to the SHM at time $t$ and $t+\mathrm{d} t$.

- $d=\|S G\|$ is the distance between the SHM and the path, which denotes the lateral error.

- $\overrightarrow{i_{s}}$ is the tangent of path $\mathscr{C}$ at $S . \overrightarrow{j_{S}}$ is perpendicular to

- $c$ denotes the curvature of the path $\mathscr{C}$, and $R$ denotes the radius of curvature at $S$, with $R=1 / c$.

- $\theta_{c}$ is the angle between the current tangent of the path and the $z$ axis, which can be considered as the direction angle of the path.

- $\theta_{e}=\theta_{d}-\theta_{c}$ defines the error of the direction angle of the SHM to the path.

- $\theta_{d}^{*}$ is the calculated target direction angle of the SHM for the control to achieve the path.

\section{B. Kinematic equations}

The aim of the path following control is to minimize the lateral and angular errors. Following [25], [26], we propose here to express the state coordinates with respect to the path $\mathscr{C}$. More precisely, the position $G$ and the direction angle $\theta_{d}$ of the SHM are described by the curvilinear coordinate $s$, the distance error $d$, and the direction angle error $\theta_{e}$. The state vector is then written as:

$$
\mathbf{q}=\left[\begin{array}{c}
s \\
d \\
\theta_{e}
\end{array}\right]
$$

The control variables of the system are the linear advance velocity and the angular velocity of the steering projected on the horizontal plane:

$$
\mathbf{u}=\left[\begin{array}{c}
v_{h} \\
\Omega_{x}
\end{array}\right]
$$

According to a simple geometry relationship, the state space model of the SHM moving on the horizontal plane is expressed as [25], [26]:

$$
\left\{\begin{array}{l}
\dot{s}=\frac{v_{h} \cos \theta_{e}}{1+d / R} \\
\dot{d}=v_{h} \sin \theta_{e} \\
\dot{\theta}_{e}=\Omega_{x}-\frac{v_{h} \cos \theta_{e}}{R+d}
\end{array}\right.
$$

\section{Control law designed with chained form}

The control objective is to ensure the convergence of the SHM to the reference path $\mathscr{C}$. Therefore, the state variables $d$ and $\theta_{e}$ are expected to be brought and kept at zero. $d=0$ means that the barycenter of the SHM belongs to path $\mathscr{C}$. $\theta_{e}=0$ ensures that the SHM will follow the path $\mathscr{C}$.

The state space model of the SHM (8) is clearly nonlinear. Samson has established in control theory that models of mobile robots can be converted into almost linear models, namely chained forms, in an exact way [27]. Such an approach is attractive and used by lots of researchers for car-like nonholonomic mobile robots [25], [26], since it allows us to use linear systems theory while still relying upon the actual nonlinear model. This approach is followed hereafter.

The model of the SHM (8) can be converted into chained form dedicated to systems with two inputs and limited to dimension three. The general chained system of dimension three is written as:

$$
\left\{\begin{array}{l}
\dot{x_{1}}=m_{1} \\
\dot{x_{2}}=x_{3} m_{1} \\
\dot{x_{3}}=m_{2}
\end{array}\right.
$$

where $\mathbf{X}=\left(x_{1}, x_{2}, x_{3}\right)^{T}$ and $\mathbf{M}=\left(m_{1}, m_{2}\right)^{T}$ are respectively the state and control vectors.

Following [26], the model of the SHM is converted into chained form by using the change of the coordinates:

$$
\begin{gathered}
x_{1}=s \\
m_{1}=\dot{x_{1}}=\frac{v_{h} \cos \theta_{e}}{1+d c} \\
x_{2}=d \\
\dot{x_{2}}=v_{h} \sin \theta_{e}=x_{3} m_{1}
\end{gathered}
$$

Therefore, from (11) and (13), the last state variable $x_{3}$ is given by:

$$
x_{3}=(1+d c) \tan \theta_{e}
$$

Finally, considering (9) and (8), the last control variable $m_{2}$ is given by:

$$
\begin{aligned}
m_{2}=\dot{x_{3}}=c v_{h} & \sin \theta_{e} \tan \theta_{e}+\frac{\mathrm{d} c}{\mathrm{~d} s} \frac{v_{h} \cos \theta_{e}}{1+d c} d \tan \theta_{e} \\
& +\frac{1+d c}{\cos ^{2} \theta_{e}}\left(\Omega_{x}-\frac{v_{h} \cos \theta_{e} c}{1+d c}\right)
\end{aligned}
$$

These transformations are invertible as long as $d \neq-\frac{1}{c}, v_{h} \neq 0$ and $\tan \theta_{e} \neq \frac{\pi}{2}[\pi]$. From a practical point of view, the SHM respects these conditions once properly initialized.

Now that the model of the SHM is converted to a linear model, the control techniques for linear systems can be applied. As shown in [26], the simple expression for the virtual control law is:

$$
\frac{m_{2}}{m_{1}}=-k_{t} x_{3}-k_{d} x_{2} \quad\left(k_{t}, k_{d}\right) \in \mathbb{R}^{+2}
$$

where $k_{t}$ and $k_{d}$ are the control parameters and are strictly positive, which implies that both $x_{2}$ and $x_{3}$ converge to 0 . In view of (12) and (14), $d$ and $\theta_{e}$ converge to 0 as well. The path following is therefore achieved. Moreover, since the evolution of the error is driven by $x_{1}=s$, the gains $\left(k_{t}, k_{d}\right)$ impose a settling distance instead of a settling time. Consequently, the helical swimmer trajectory will be identical if the initial error is the same. The guidance performances will be velocity independent, which are suitable for low Reynolds numbers environments.

The control law on the angular velocity of the steering of the SHM is expressed as follows, by reporting (11) and (15) into (16): 


$$
\begin{array}{r}
\Omega_{x}=-\frac{\cos ^{3} \theta_{e}}{1+d c}\left(k_{d} d+k_{t}(1+d c) \tan \theta_{e}+\frac{\mathrm{d} c}{\mathrm{~d} s} d \tan \theta_{e}\right. \\
\left.+c \tan ^{2} \theta_{e}(1+d c)\right)+\frac{v_{h} \cos \theta_{e}}{R+d}
\end{array}
$$

where $v_{h}$ is the advance velocity projected on the horizontal plane, which is usually constant, and depends on the frequency of the rotating field and the inclination angle. In the case where the reference path is a straight line, the control of the angular speed of the steering projected on the horizontal plane can be simplified as:

$$
\Omega_{x}=-\cos ^{3} \theta_{e}\left(k_{d} d+k_{t} \tan \theta_{e}\right)
$$

The target direction angle can be calculated by the real-time direction angle and the angular velocity of the steering of the SHM, which can be expressed as:

$$
\theta_{d}^{*}(t)=\theta_{d}(t)+\Omega_{x} \mathrm{~d} t
$$

The target orientation of the SHM $\mathbf{n}^{*}$ can be calculated with the target direction angle $\theta_{d}^{*}$ and target inclination angle $\theta_{i}^{*}$ with (3). The target inclination angle is fixed for many tasks.

The path following problem is now converted into a 3D steering problem of the SHM from the real-time orientation $\mathbf{n}$ to a target orientation $\mathbf{n}^{*}$. The control of the magnetic field which enables the steering of the SHM from $\mathbf{n}$ to $\mathbf{n}^{*}$ will be studied in the next section.

\section{3D STEERING CONTROL}

\section{A. Modelling of steering}

The magnetization of the helical swimmer is noted as $\mathbf{M}$, as shown in Fig. 3. The magnetic torque $\tau$ exerted on the helical swimmer can be expressed as :

$$
\tau=\mathbf{M} \times \mathbf{B}
$$

The magnetic field $\mathbf{B}$ can be decomposed in the directions perpendicular to its axis $\mathbf{B}_{\perp \mathbf{n}}$, and colinear to its axis $\mathbf{B}_{\| \mathbf{n}}$. As the magnetization of the helical swimmer is always perpendicular to its own axis $\mathbf{n}$, the magnetic torque can also be decomposed in the two directions:

$$
\tau=\underbrace{\mathbf{M} \times \mathbf{B}_{\perp \mathbf{n}}}_{\tau_{\| \mathbf{n}}}+\underbrace{\mathbf{M} \times \mathbf{B}_{\| \mathbf{n}}}_{\tau_{\perp \mathbf{n}}}
$$

The torque $\tau_{\| \mathbf{n}}$ colinear to $\mathbf{n}$ makes the helical swimmer rotate around its current axis. The torque $\tau_{\perp \mathbf{n}}$ perpendicular to $\mathbf{n}$ makes the helical swimmer rotate around $\mathbf{M} \times \mathbf{n}$. It means that the axis of the helical swimmer changes the direction, which enables the 3D steering of the helical swimmer.

\section{B. Self-rotation control}

The magnetic field $\mathbf{B}_{\perp \mathbf{n}}$ rotating around the axis $\mathbf{n}$ can be expressed as:

$$
\mathbf{B}_{\perp \mathbf{n}}=B_{0} \cos (2 \pi f t) \tilde{\mathbf{u}}+B_{0} \cos (2 \pi f t) \tilde{\mathbf{v}}
$$

where $B_{0}$ is the magnetic flux density at the center of the Helmholtz coils, $f$ is the rotation frequency, and $(\tilde{\mathbf{u}}, \tilde{\mathbf{v}})$ are the basis vectors of the plane orthogonal to the axis $\mathbf{n}$ :

$$
\begin{aligned}
& \tilde{\mathbf{u}}=\frac{\mathbf{u}}{\|\mathbf{u}\|}=\frac{1}{\|\mathbf{u}\|}\left[\begin{array}{c}
n_{y} \\
-n_{x} \\
0
\end{array}\right] \\
& \tilde{\mathbf{v}}=\frac{\mathbf{v}}{\|\mathbf{v}\|}=\frac{1}{\|\mathbf{v}\|}\left[\begin{array}{c}
n_{x} n_{z} \\
n_{y} n_{z} \\
-n_{x}^{2}-n_{y}^{2}
\end{array}\right]
\end{aligned}
$$

with $n_{x} \neq 0$ or $n_{y} \neq 0$. Remark that if $n_{x}=0$ and $n_{y}=0$, we can chose $\tilde{\mathbf{u}}=(1,0,0)^{T}$ and $\tilde{\mathbf{v}}=(0,1,0)^{T}$.

\section{Steering control}

The magnetic field $\mathbf{B}_{\| \mathbf{n}}$, which contributes to the steering, depends on the orientation error of the SHM. The orientation error is defined as the sine of the angle between the realtime orientation and the target orientation: $\sin \left(\mathbf{n}, \mathbf{n}^{*}\right)$, which is equivalent to $\left\|\mathbf{n} \times \mathbf{n}^{*}\right\|$.

Let us define a control proportional to this error. As shown in Fig. 5, the direction of $\mathbf{B}_{\| \mathbf{n}}$ depends on the projection of $\mathbf{B}_{\perp \mathbf{n}}$ on the target orientation $\mathbf{n}^{*}$. If this projection is positive $\mathbf{B}_{\perp \mathbf{n}} \cdot \mathbf{n}^{*}>0$, the magnetic field $\mathbf{B}_{\| \mathbf{n}}$ should be in the direction of $-\mathbf{n}$. If this projection is negative, $\mathbf{B}_{\| \mathbf{n}}$ should be in the same direction as $\mathbf{n}$.

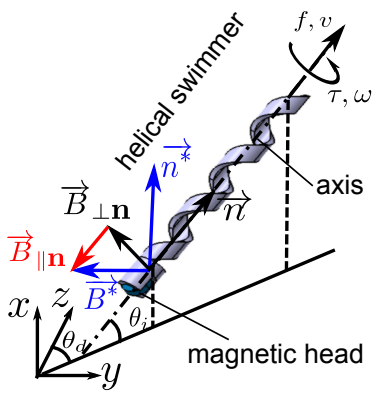

(a) $\mathbf{B}_{\perp \mathbf{n}} \cdot \mathbf{n}^{*}>0$

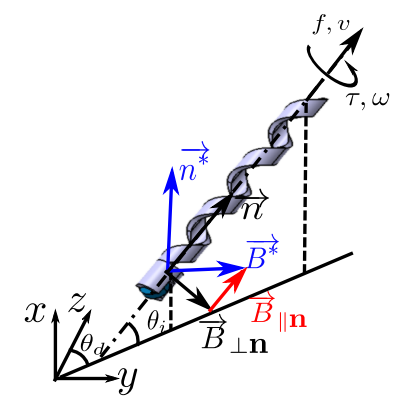

(b) $\mathbf{B}_{\perp \mathbf{n}} \cdot \mathbf{n}^{*}<0$
Fig. 5: The direction of the magnetic field $\mathbf{B}_{\| \mathbf{n}}$ depends on the projection of $\mathbf{B}_{\perp \mathbf{n}}$ on the target orientation of the SHM $\mathbf{n}^{*}$ : (a) along $\mathbf{n}^{*}$, and (b) along $-\mathbf{n}^{*}$.

Therefore, the control law of $\mathbf{B}_{\| \mathbf{n}}$ can be expressed as:

$$
\mathbf{B}_{\| \mathbf{n}}=-\operatorname{sign}\left(\mathbf{B}_{\perp \mathbf{n}} \cdot \mathbf{n}^{*}\right) \cdot \lambda\left\|\mathbf{n} \times \mathbf{n}^{*}\right\| \mathbf{n}
$$

with $\lambda$ the control parameter.

In conclusion, the control law of the magnetic field $\mathbf{B}$ which enables the 3D steering of helical swimmers can be expressed as:

$$
\mathbf{B}=\mathbf{B}_{\perp \mathbf{n}}+\mathbf{B}_{\| \mathbf{n}}
$$

by replacing $\mathbf{B}_{\perp \mathbf{n}}$ by (22) and $\mathbf{B}_{\| \mathbf{n}}$ by (25). The input of the system is the target orientation $\mathbf{n}^{*}$. The real-time orientation of the SHM $\mathbf{n}$ can be constructed from the real-time tracking on the images, which will be introduced in Section VII. 


\section{SySTEM OVERVIEW}

\section{A. Magnetic actuation and stereo vision system}

The SHM in this paper is actuated by a 3D Helmholtz coils system. Three Helmholtz coil pairs are orthogonally arranged to generate a uniform rotating field in the $3 \mathrm{D}$ space. The 3D world coordinate system $\mathscr{R}_{w}=(O, x, y, z)$ is defined in Fig. 6a: $x, y, z$ are respectively the axis of the small, medium, and big coils. The maximum magnetic flux density is designed as $10 \mathrm{mT}$. Each pair is driven by a Maxon ADS 50/5 4-Q-DC servoamplifier, capable of $5 \mathrm{~A}$ continuous current. Analog communication between the PC and the amplifiers is accomplished with a Sensoray 626 Analog and Digital I/O card. The magnetic fields generated by the three coil pairs were measured and calibrated by a gaussmeter Hirst GM08.

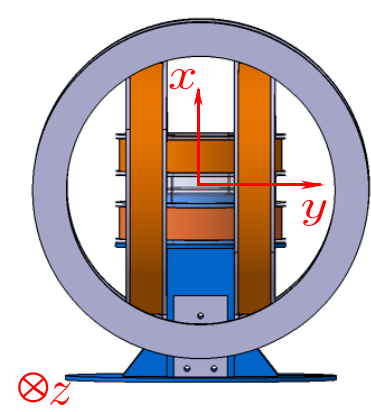

(a)

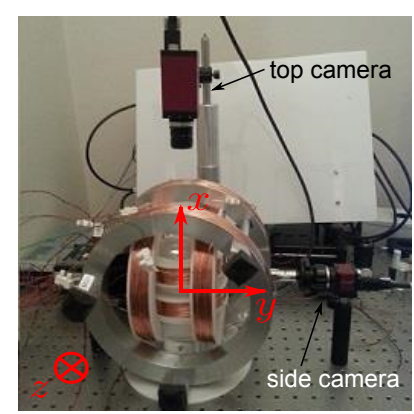

(b)
Fig. 6: (a) Three orthogonal Helmholtz coil pairs CAD design. (b) Experimental setup: Helmholtz coils, top camera, side camera with endoscope.

The stereo vision system consists of two firewire cameras, as shown in Fig. 6b: one is on the top (Pike F032B) of, the other is beside the Helmholtz coils system (Guppy Pro F032). Due to the limited side opening, an endoscope (Bipol) with diameter of $2.7 \mathrm{~mm}$ is mounted on the camera instead of a simple lens.

\section{B. Helical swimmer at low Reynolds number}

We designed a Scaled-up Helical Swimmer (SHM) with a magnetic head, as shown in Fig. 7a, and as presented in [28]. The SHM is painted in black in order to have a good image contrast for tracking. It has an overall length of $17.8 \mathrm{~mm}$ with 3.5 turns, and a diameter of $1 \mathrm{~mm}$. The width of the helical tail is $1.4 \mathrm{~mm}$, and the thickness is $0.3 \mathrm{~mm}$. A permanent magnet is planted in the head. The magnet is magnetized in the direction of the diameter, so that the SHM will rotate around its own axis in a rotating magnetic field.

Helical swimmers with a magnetic head show a cut-off frequency [8]: below the cut-off frequency, they rotate in sync with the external rotating magnetic field; beyond this cut-off frequency, their rotation frequency decreases. The cut-off frequency depends on the Reynolds number. In the following experiments, the SHM is put in pure glycerol liquid. The glycerol density measured by a portable density meter (DMA 35) is $1.26 \times 10^{3} \mathrm{~kg} / \mathrm{m}^{3}$. The viscosity of glycerol measured by a falling ball viscometer (Brookfield KF10) is

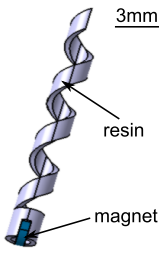

(a)

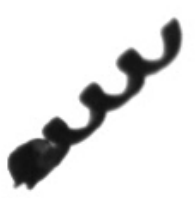

(b)

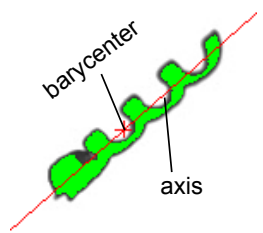

(c)
Fig. 7: (a) CAD design of the SHM with a magnetic head. (b) The fabricated SHM. (c) The SHM is real-time tracked by ViSP [29]. The red cross is the barycentre and the red line is the axis.

$1350 \mathrm{mPa} \cdot \mathrm{s}$ at $23^{\circ} \mathrm{C}$. The $\mathrm{SHM}$ swims at $0.5 \mathrm{~mm} / \mathrm{s}-1 \mathrm{~mm} / \mathrm{s}$ in the experiments. The calculated Reynolds numbers are thus $0.0005-0.001$. In water, a swimming bacterium such as E.coli has a $R e \approx 10^{-5}-10^{-4}$, and a human spermatozoon moves with $R e \approx 0.01$ [30]. The cut-off frequency of the used SHM in pure glycerol is measured as $5 \mathrm{~Hz}$. The rotation frequency of the magnetic field is always below the cut-off frequency in the following experiments, so that the SHM is supposed to always rotate synchronously with the magnetic field.

\section{Real-time tracking on the image}

A robust real-time tracking of the SHM is crucial for visual servoing tasks, which is realized by ViSP [29]. The image of the SHM is considered as a blob (vpDot class in ViSP) defined by a set of connected pixels with the same gray level. The image of the SHM is shown in Fig. 7b, and tracked blob is shown in Fig. 7c and presented in green.

The barycentre and the axis of a helical swimmer can be calculated from image moments [31]. The barycenter of the SHM is presented in Fig. 7c by a red cross. The axis of a SHM in the image can be defined by the direction of the axis of least inertia. The axis of the SHM is presented in Fig. 7c by a red line. The 3D pose of the SHM can be estimated based on the barycenter and the axis tracked from the images, which will be presented in the next section.

\section{REAL-TIME 3D POSE ESTIMATION}

The 3D pose of the SHM is described by its orientation and its position, which are respectively defined as the normal vector of the real-time axis and barycenter of the SHM in the 3D space. The methods to estimate the orientation and the position of the SHM, using the axes and the barycenters tracked from the two images taken with the top and side cameras, will be introduced hereafter. The calibration of the cameras are detailed in [32].

\section{A. Real-time orientation estimation}

The axis of the SHM in the 3D space is the intersection of the two planes $\mathscr{P}_{t}$ and $\mathscr{P}_{s}$, which are defined by the projection centers of the cameras and the axis of the SHM $\mathbf{n}$, as shown in Fig. 8. It can then be calculated by the vector product of the two normal vectors $\mathbf{h}_{t}$ and $\mathbf{h}_{s}$ of the two planes [33]. 


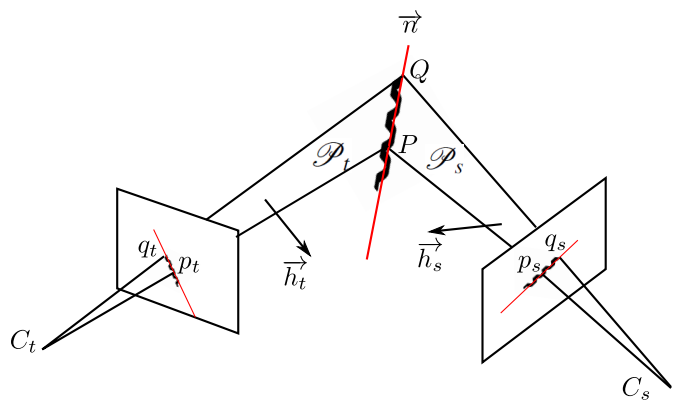

Fig. 8: Determination of the axis of the SHM in the world coordinate system.

The point $P$ is a point on the axis of the SHM, with ${ }^{c t} \mathbf{P}=$ $\left({ }^{c t} X,{ }^{c t} Y,{ }^{c t} Z\right)^{T}$ in the top camera coordinate system. As $P$ is in the plane $\mathscr{P}_{t}$, we can write:

$$
h_{t x}{ }^{c t} X+h_{t y}{ }^{c t} Y+h_{t z}{ }^{c t} Z=0
$$

with ${ }^{c t} \mathbf{h}_{t}=\left(h_{t x}, h_{t y}, h_{t z}\right)^{T}$ the normal vector $\mathbf{h}_{t}$ in the top camera coordinate system. Then,

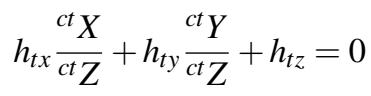

The point $p_{t}$ is the image of the point $P$, with ${ }^{i m} \mathbf{p}_{t}=$ $\left(\frac{{ }^{c t} X}{{ }^{c t} Z}, \frac{{ }^{c t} Y}{{ }^{c t} Z}\right)^{T}$ in the metric image plane of the top camera by definition, and ${ }^{i m} \tilde{\mathbf{p}}_{t}$ its homogeneous coordinates. The relationship (28) defining the equation of the line in the image supporting the helical swimmer can be written under a matrix form:

$$
{ }^{c t} \mathbf{h}_{t}^{T}{ }^{i m t} \tilde{\mathbf{p}_{t}}=0
$$

with ${ }^{i m t} \tilde{\mathbf{p}_{t}}$ obtained by measuring the point $p_{t}$ in the pixel image plane of the top camera. Then, with $\left\|\mathbf{h}_{t}\right\|=1$, we can obtain ${ }^{c t} \mathbf{h}_{t}$.

The unit normal vector $\mathbf{h}_{s}$ of the plane $\mathscr{P}_{s}$ in the side camera coordinate system ${ }^{c s} \mathbf{h}_{s}$ can be obtained by the same method. Therefore, the axis of the SHM in the top camera coordinate system can be expressed as:

$$
{ }^{c t} \mathbf{n}={ }^{c t} \mathbf{h}_{t} \times{ }^{c t} \mathbf{h}_{s}={ }^{c t} \mathbf{h}_{t} \times\left({ }^{c t} \mathbf{R}_{c s}{ }^{c s} \mathbf{h}_{s}\right)
$$

where ${ }^{c t} \mathbf{R}_{c s}$ is the rotation matrix from the side camera coordinate system to the top camera coordinate system.

Then, the axis of the SHM in 3D world coordinate system can be written as:

$$
\mathbf{n}={ }^{w} \mathbf{R}_{c t}{ }^{c t} \mathbf{n}
$$

where ${ }^{w} \mathbf{R}_{c t}$ is the rotation matrix from the top camera coordinate system to the world coordinate system. The orientation of the SHM is then obtained.

\section{B. Real-time position estimation}

The real-time position of the SHM is estimated by triangulation [34]. Based on the projective model of the camera, the images of the barycenter of the SHM on the images planes are linearly dependent with it. Therefore, their cross products are zero, with the transformation between the two camera coordinate systems, we can write:

$$
\left\{\begin{array}{l}
{ }^{i m t} \tilde{\mathbf{g}_{t}} \times\left({ }^{c t} \mathbf{R}_{c s}{ }^{c s} \mathbf{G}\right)+{ }^{i m t} \tilde{\mathbf{g}_{t}} \times{ }^{c t} \mathbf{t}_{c s}=\mathbf{0} \\
{ }^{i m s} \tilde{\mathbf{g}_{s}} \times{ }^{c s} \mathbf{G}=\mathbf{0}
\end{array}\right.
$$

where ${ }^{c t} \mathbf{R}_{c s}$ and ${ }^{c t} \mathbf{t}_{c s}$ are respectively the rotation and translation matrix from the side camera to the top camera coordinate system. The system can be solved with the Singular Value Decomposition (SVD) method to get the point $\mathrm{G}$ in the side camera coordinate system ${ }^{c s} \mathbf{G}$. Then, the barycenter $G$ of the SHM in the 3D world coordinate system can be expressed as:

$$
{ }^{w} \mathbf{G}={ }^{w} \mathbf{R}_{c s}{ }^{c s} \mathbf{G}+{ }^{w} \mathbf{t}_{c s}
$$

where ${ }^{w} \mathbf{R}_{c s}$ and ${ }^{w} \mathbf{t}_{c s}$ are respectively the rotation and translation matrix from the side camera to the world coordinate system. The position of the barycenter of the SHM is then obtained.

Once the real-time orientation and position of the SHM are obtained, the control loop is closed, as shown in Fig. 2. According to our best knowledge, the control law of the magnetic field actuating a helical swimmer to follow a reference path with visual feedback is proposed for the first time.

\section{RESULTS}

\section{A. Control parameter of the $3 D$ steering}

First of all, the control parameter of the 3D steering should be determined. The magnetic field is controlled by the output voltage of the PCI card $U$. As $U$ is proportional to $B, U$ can be written in the same form as (25) with a control parameter of $\lambda_{U}$. According to the maximum capacity of the Helmholtz setup, the control parameter $\lambda_{U}$ is limited to 3 . The performances of the 3D steering of the SHM with different control parameters are shown in Fig. 9, with an initial position of $0 / 40$ (direction angle / inclination angle) to a target position of $60 / 40$. The rotation frequency is $2 \mathrm{~Hz}$. Note that an oscillation of the calculated real-time angle of the SHM is observed, which is due to the asymmetry of the helical geometry.

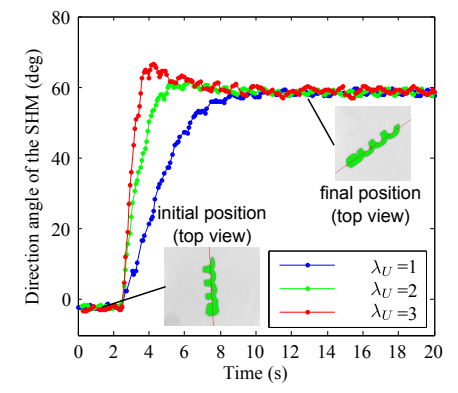

(a)

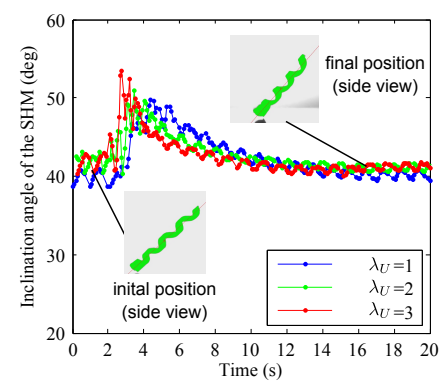

(b)
Fig. 9: Closed-loop responses of the real-time (a) direction angle and (b) inclination angle of the SHM to the 3D steering with different control parameters.

The control parameter is chosen as $\lambda_{U}=2$, because of the response time and reasonable overshooting, as shown in Fig. 9a. An impulse of the inclination angle is observed in 
Fig. 9b, although the target inclination angle is the same as the initial inclination angle. The inclination angle increases more with a higher control parameter, because the magnetic torque exerted on the SHM is higher.

The trajectory of the SHM for the 3D steering from $0 / 40$ to $60 / 40$ is traced on a unit sphere, as shown in Fig. 10. The movements are described in the reference frame relative to the center of the head of SHM. Static errors between the target orientation and the final orientation can be observed on the figure. As these errors are the same for different parameters, which are about $-2^{\circ}(3 \%)$, we presume that these errors are due to the calibration error of the system.

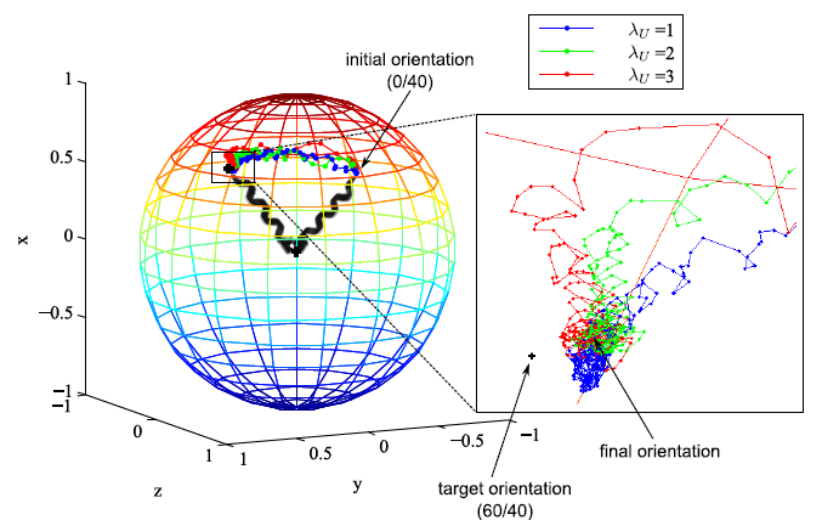

Fig. 10: Trajectories of the SHM for 3D steering control with different control parameters.

Once the control parameter is determined, visual servo control for 3D steering of the SHM will be tested with more command angles.

\section{B. Visual servo control for $3 D$ steering}

The 3D steering with zero angle is firstly validated. There is no initial error, the SHM stabilizes at the initial orientation. The control of the $3 \mathrm{D}$ steering is sensitve to small angle control, which is validated with an initial error of $5^{\circ}$. The steering of direction angles are then tested every $60^{\circ}$ in a turn, with an inclination angle of $40^{\circ}$. The errors in direction angle during the steering are shown in Fig. 11. The convergence of the errors states that the steering is achieved.

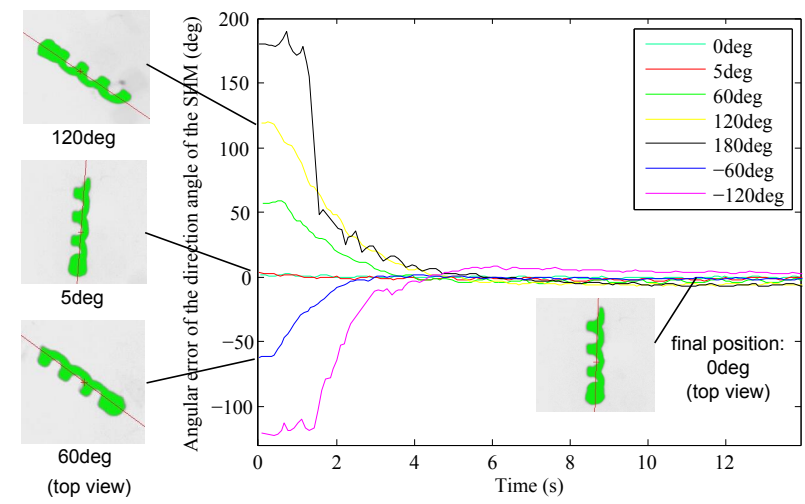

Fig. 11: Error of the orientation during the 3D steering control.
The trajectories are traced on the unit sphere, as shown in Fig. 12. Note that when steering at negative direction angles, such as $-60^{\circ}$, the inclination decreases and then increases during the control, which is contrary to steering at a positive direction angle. This is due to the direction of the self-rotation of the SHM.

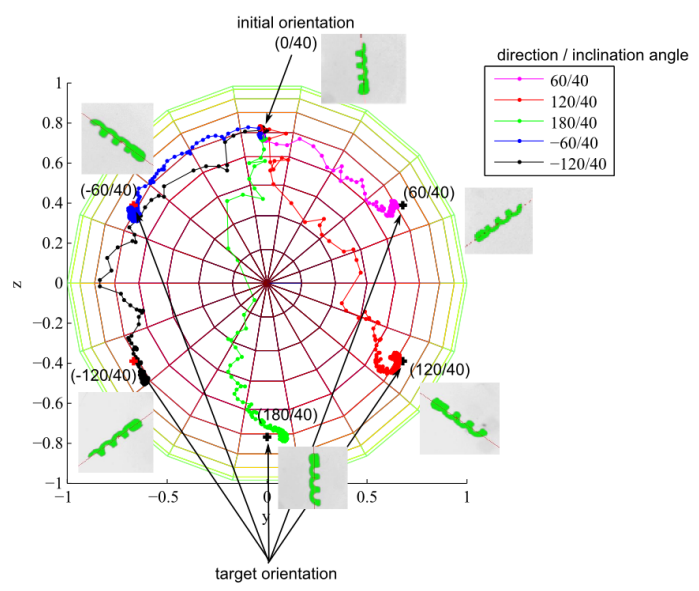

Fig. 12: Trajectories of the SHM for the 3D steering control with different direction command angles.

Hereafter, the 3D steering control is achieved with the same target direction angle and different target inclination angles. The trajectories are traced in Fig. 13.

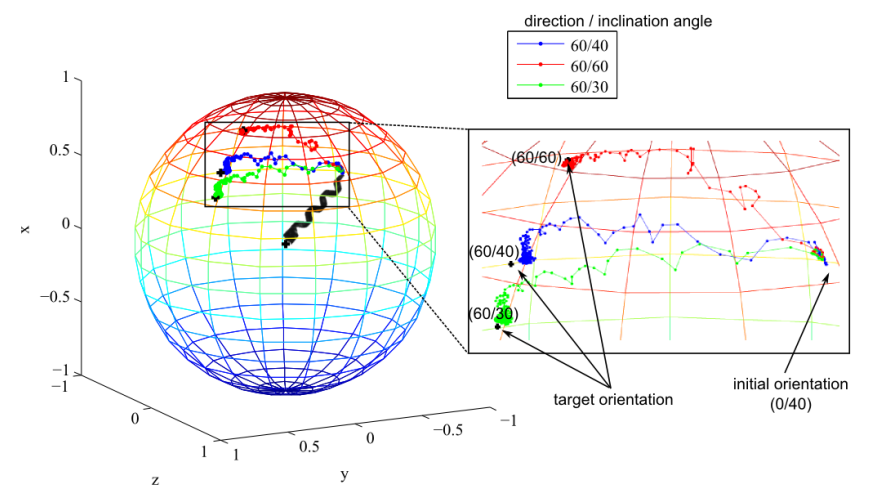

Fig. 13: Trajectories of the SHM for the 3D steering control with different inclination command angles.

The visual servo control for the 3D steering of the SHM in the 3D space is validated. According to our best knowledge, the closed-loop control of the 3D steering of helical swimmers is realized for the first time. The closed-loop 3D steering control of helical swimmers is crucial to realize a controlled $3 \mathrm{D}$ motion.

\section{Visual servo control of the planar path following}

The visual servo control for the path following of the SHM was tested with a reference path of a straight line on the horizontal plane. The inclination angle of the SHM is kept at $40^{\circ}$ empirically to maintain its altitude. The advancing velocity of the SHM is measured as $0.48 \mathrm{~mm} / \mathrm{s}$ with $3 \mathrm{~Hz}$. Let us consider the straight line of $y=0$. The SHM is 
initially off the path, with an initial distance error of about $-5 \mathrm{~mm}$. Trajectories of the barycenter of the SHM during path following control with different control parameters are traced on Fig. 14.

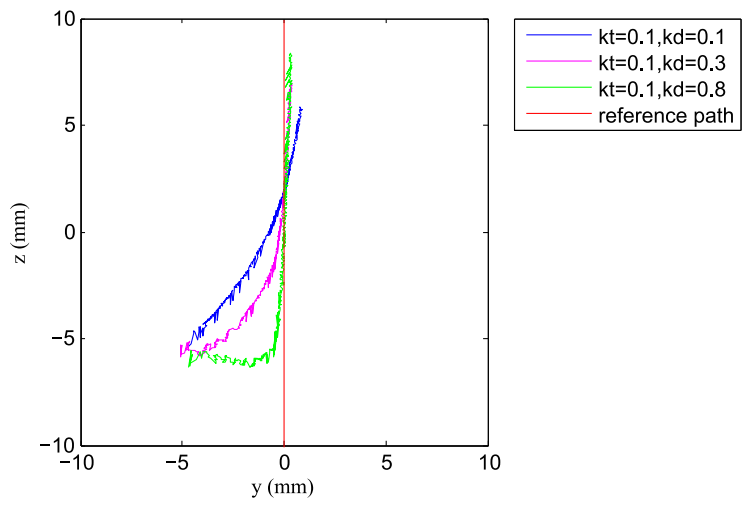

Fig. 14: Trajectories of the SHM during visual servo control for path following of a straight line with different parameters.

The parameter $k_{t}$ is for the direction angle error, and the parameter $k_{d}$ is for the distance error. For the same $k_{t}$, the travelled path of the SHM is the shortest with the least $k_{d}$; and the SHM reaches the reference path the fastest with the highest $k_{d}$. Therefore, the choice of the control parameters depends on the requirement of the system: reaching the reference quickly or travelling less.

An image sequence of the SHM during the visual servo control for the path following of a straight line is shown in Fig. 15. The reference path is presented by a blue line. The SHM reached the reference path, then went along this reference path. In fact, the SHM presents drifting in the $y$ axis due to the boundary effect [17]. As the control scheme takes into account both the orientation error and the position error, the closed-loop control corrects this distance error with a negative target direction angle. Therefore, a propulsive force is generated in the $-y$ axis to counterbalance this drifting effect. An open-loop control for advancing along the line of $y=0$ axis will be compared in the next section.

\section{Open-loop vs closed-loop}

The trajectory of the SHM for an open-loop control of following a straight line of $y=0$ is traced on Fig. 16, as well as a trajectory for a visual servo control of following the same reference path without initial distance error. A perturbation of the force in the direction of $y$ on the SHM is observed during the open-loop control, due to the friction with the substrate. In case of the closed-loop control by visual servoing, the SHM returned onto the reference line despite the perturbation. We define that the SHM is on the path while the distance between the SHM and the path is $<0.5 \mathrm{~mm}$. Then, for the visual servo path following, the SHM is always on the path. As for the open-loop control, the SHM is off the path from about $15 \mathrm{~s}$ and never goes back onto the path. Therefore, visual servo control of SHM shows more precise motion than a simple open-loop control.

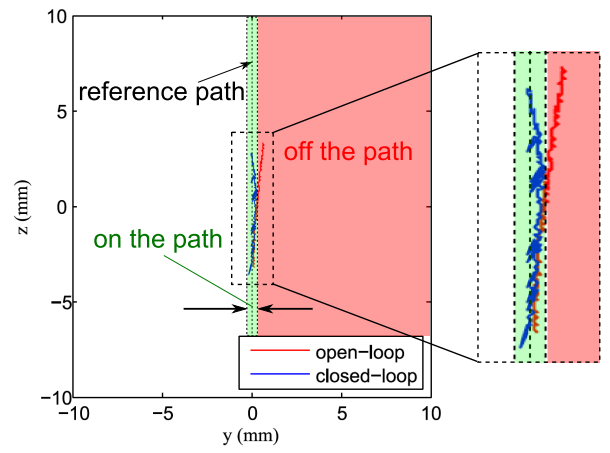

Fig. 16: Comparison of the trajectories of the open-loop control and visual servo control for following a straight line of $y=0$.

According to our best knowledge, the closed-loop control for the path following of helical swimmers is realized for the first time.

\section{CONCLUSION AND PERSPECTIVES}

In this paper, we proposed a control law of the rotating magnetic field for planar path following of helical swimmers. The helical swimmer is millimeter-scaled in a viscous liquid to simulate the low Reynolds number environment. A 3D pose estimation including the position and orientation of helical swimmers is based on the real-time tracking on the images taken by a stereo vision system. The results of the experiments show that the helical swimmer enables closedloop 3D steering by visual servoing for the first time according to our knowledge. The planar path following of a straight line was also achieved. The control parameters define the trajectories of the helical swimmer: reaching the reference path quickly or traveling less. In future works, we will study the 3D path following of helical swimmers. Then, we will scale the helical swimmers down to the microscale, and apply the same control method with suitable optical devices. We expect that helical swimmers will enable closed-loop 3D motion in the future.

\section{ACKNOWLEDGMENT}

We acknowledge funding from Émergence-UPMC-2012 research program, the Franche-Comté region, and ACTION, the French ANR Labex no. ANR-11-LABX-01-01. The authors are also grateful to Sinan Haliyo, Sylvain Pledel, Christophe Grand, Thomas Seon, Tianyi Li and Jean-ochin Abrahamians for providing technical supports on the expermental setup.

\section{REFERENCES}

[1] N. Chaillet and S. Régnier, Microrobotics for Micromanipulation. ISTE, Wiley, 2013.

[2] B. Nelson, I. Kaliakatsos, and J. Abbott, "Microrobots for minimally invasive medicine," Annual Review of Biomedical Engineering, vol. 12, no. 1, pp. 55-85, 2010.

[3] E. Purcell, "Life at low reynolds number.," American Journal of Physics, vol. 45, no. 1, pp. 3-11, 1977.

[4] E. Purcell, "The efficiency of propulsion by a rotating flagellum.," Proc Natl Acad Sci U S A, vol. 94, no. 21, pp. 11307-11, 1997. 


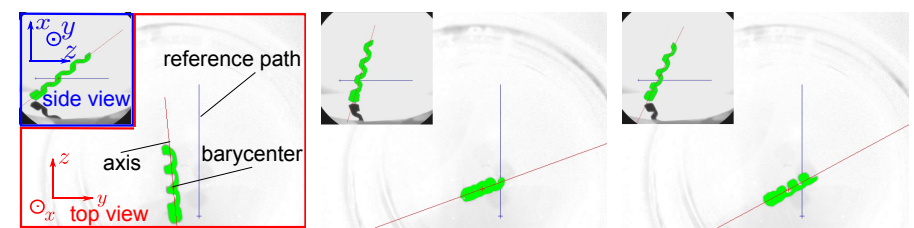

(a) $0 \mathrm{~s}$ (b) $1 \mathrm{~s}$

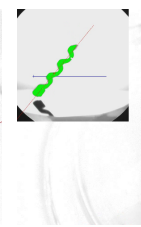

(d) $3 \mathrm{~s}$

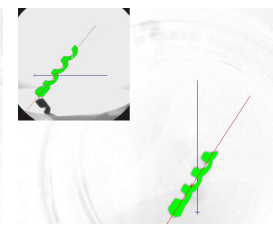

(e) $5 \mathrm{~s}$

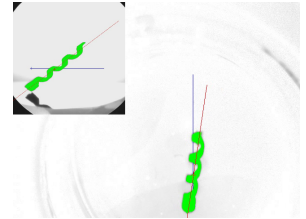

(f) $6 \mathrm{~s}$

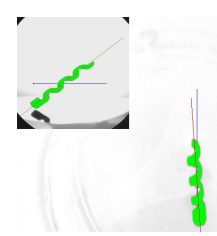

(g) $7 \mathrm{~s}$

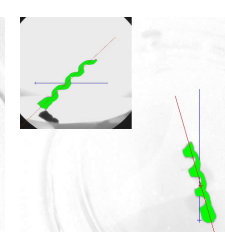

(h) $8 \mathrm{~s}$

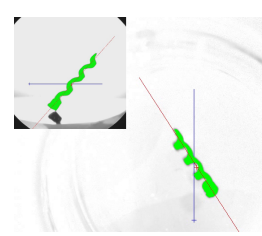

(i) $11 \mathrm{~s}$

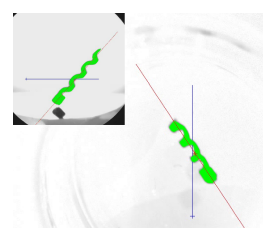

(j) $13 \mathrm{~s}$

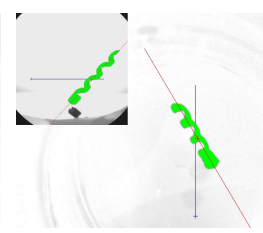

(k) $16 \mathrm{~s}$

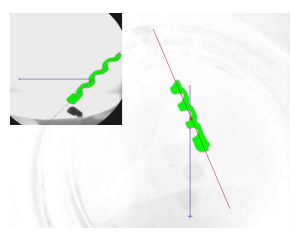

(1) $19 \mathrm{~s}$

Fig. 15: Image sequence of the SHM during the visual servo control for path following of a straight line with the control parameters of $k_{t}=0.1$ and $k_{d}=0.8$ taken by the top camera, and the side camera (insert at the top-left corner). The image sequence demonstrates that thanks to the control scheme, the barycenter of the SHM can reach the reference path and then follow it.

[5] H. Berg and R. Anderson, "Bacteria swim by rotating their flagellar filaments," Nature, vol. 245, no. 5425, pp. 380-382, 1973.

[6] T. Honda, K. Arai, and K. Ishiyama, "Micro swimming mechanisms propelled by external magnetic fields," Magnetics, IEEE Transactions on, vol. 32, pp. $5085-5087$, sep 1996.

[7] K. Ishiyama, K. Arai, M. Sendoh, and A. Yamazaki, "Spiral-type micro-machine for medical applications," in Micromechatronics and Human Science, 2000. MHS 2000. Proceedings of 2000 International Symposium on, pp. $65-69,2000$.

[8] T. Xu, G. Hwang, N. Andreff, and S. Regnier, "Modeling and swimming property characterizations of scaled-up helical microswimmers," Mechatronics, IEEE/ASME Transactions on, vol. 19, no. 3, pp. 10691079, 2014.

[9] D. Bell, L. Dong, B. Nelson, M. Golling, L. Zhang, and D. Grutzmacher, "Fabrication and characterization of three-dimensional ingaas/gaas nanosprings," Nano Letters, vol. 6, no. 4, pp. pp. 725-729, 2006.

[10] L. Zhang, J. Abbott, L. Dong, B. Kratochvil, D. Bell, and B. Nelson, "Artificial bacterial flagella: Fabrication and magnetic control," Appl. Phys. Lett., vol. 94, no. 6, 2009.

[11] G. Hwang, R. Braive, L. Couraud, A. Cavanna, O. Abdelkarim, I. Robert-Philip, A. Beveratos, I. Sagnes, S. Haliyo, and S. Régnier, "Electro-osmotic propulsion of helical nanobelt swimmers," The International Journal of Robotics Research, vol. 30, no. 7, pp. 806-819, 2011.

[12] L. Pooyath, R. Sai, Y. Chandorkar, B. Basu, S. Shivashankar, and A. Ghosh, "Conformal cytocompatible ferrite coatings facilitate the realization of a nano-voyager in human blood.," Nano letters, 2014.

[13] A. Ghosh and P. Fischer, "Controlled propulsion of artificial magnetic nanostructured propellers," Nano Letters, vol. 9, no. 6, pp. 2243-2245, 2009.

[14] A. Mahoney, J. Sarrazin, E. Bamberg, and J. Abbott, "Velocity control with gravity compensation for magnetic helical microswimmers.," Advanced Robotics, vol. 25, no. 8, pp. 1007-1028, 2011.

[15] S. Tottori, L. Zhang, F. Qiu, K. Krawczyk, A. Franco-Obregn, and B. Nelson, "Magnetic helical micromachines: Fabrication, controlled swimming, and cargo transport," Advanced Materials, vol. 24, pp. pp. 811-816, February 2012. highlighted as the front cover.

[16] A. Ghosh, D. Paria, G. Rangarajan, and A. Ghosh, "Velocity fluctuations in helical propulsion: How small can a propeller be," The Journal of Physical Chemistry Letters, vol. 5, no. 1, pp. 62-68, 2014.

[17] K. Peyer, L. Zhang, B. Kratochvil, and B. Nelson, "Non-ideal swimming of artificial bacterial flagella near a surface," in Robotics and Automation (ICRA), 2010 IEEE International Conference on, pp. 96-101, May 2010.

[18] J. A. Gangloff and M. F. de Mathelin, "High-speed visual servoing of a 6-dof manipulator using multivariable predictive control," Advanced Robotics, vol. 17, no. 10, pp. 993-1021, 2003.

[19] R. Dahmouche, N. Andreff, Y. Mezouar, O. Ait-Aider, and P. Martinet "Dynamic visual servoing from sequential regions of interest acquisi- tion," The International Journal of Robotics Research, vol. 31, no. 4, pp. 520-537, 2012.

[20] J. Courbon, Y. Mezouar, N. Guénard, and P. Martinet, "Vision-based navigation of unmanned aerial vehicles," Control Engineering Practice, vol. 18 , no. 7, pp. 789-799, 2010.

[21] A. Bloch, J. Marsden, and D. Zenkov, "Nonholonomic dynamics," in Control of Nonholonomic Systems on Riemannian Manifolds. PROCEEDINGS OF NOLCOS 92, 2005.

[22] D. Jiles, Introduction to magnetism and magnetic materials; 2nd ed. Boca Raton, FL: Chapman and Hall, 1998.

[23] A. Cherubini, F. Chaumette, and G. Oriolo, "A position-based visual servoing scheme for following paths with nonholonomic mobile robots," in Intelligent Robots and Systems, 2008. IROS 2008. IEEE/RSJ International Conference on, pp. $1648-1654$, sept. 2008.

[24] A. Cherubini, F. Chaumette, and G. Oriolo, "An image-based visual servoing scheme for following paths with nonholonomic mobile robots," in Control, Automation, Robotics and Vision, 2008. ICARCV 2008. 10th International Conference on, pp. 108 -113, dec. 2008.

[25] J. Laumond, ed., La robotique mobile. Traité IC2, Paris: Hermés science, 2001.

[26] B. Thuilot, C. Cariou, P. Martinet, and M. Berducat, "Automatic guidance of a farm tractor relying on a single cp-dgps," Auton. Robots, vol. 13, pp. 53-71, July 2002.

[27] C. Samson, "Control of chained systems application to path following and time-varying point-stabilization of mobile robots," Automatic Control, IEEE Transactions on, vol. 40, no. 1, pp. 64-77, 1995.

[28] T. Xu, G. Hwang, N. Andreff, and S. Régnier, "Characterization of three-dimensional steering for helical swimmers," in ICRA'14 IEEE International Conference on Robotics and Automation, pp. 4045 - 4051, 2014.

[29] E. Marchand, F. Spindler, and F. Chaumette, "Visp for visual servoing: a generic software platform with a wide class of robot control skills," Robotics Automation Magazine, IEEE, vol. 12, pp. 40 -52, dec. 2005.

[30] E. Lauga and T. Powers, "The hydrodynamics of swimming microorganisms," Reports on Progress in Physics, vol. 72, no. 9, p. 096601, 2009.

[31] B. Horn, Robot Vision. The MIT Press McGraw-Hill Higher Education, 1st ed., 1986.

[32] T. Xu, Propulsion Characteristics and Visual Servo Control of Scaled-up Helical Microswimmers. PhD thesis, Université Pierre et Marie CurieParis VI, 2014.

[33] N. Andreff, B. Espiau, and R. Horaud, "Visual servoing from lines," The International Journal of Robotics Research, vol. 21, no. 8, pp. 679-699, 2002.

[34] R. I. Hartley and A. Zisserman, Multiple View Geometry in Computer Vision. Cambridge University Press, ISBN: 0521540518, second ed., 2004. 


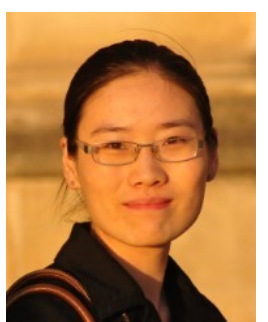

microswimmers.

Tiantian Xu received the M.S. degree in Industrial Engineering from the Ecole Centrale Paris, France, the Engineer degree ( M.S. degree) in Mechanic from Supmeca, France, in 2010, and the Ph.D. degree at the Institute of Intelligent Systems and Robotics (ISIR), University of Pierre and Marie Curie, Paris, France, in 2013.

She is currently working in the Chinese University of Hong Kong, in the department of Mechanical and Automation Engineering. Her research interests are currently focused on design and control of helical

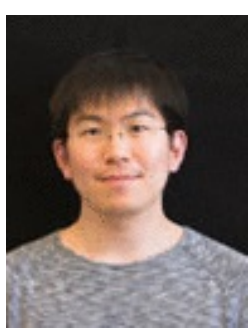

Gilgueng Hwang obtained both M.Sc. and Ph.D degrees of Electrical Engineering from University of Tokyo in 2005 and 2008 respectively. Part of this $\mathrm{Ph} . \mathrm{D}$ work was done by the collaboration with Prof. Bradley J. Nelson's multiscale robotics group in the Institute of Robotics and Intelligent Systems (IRIS), ETHZ, Switzerland. He has worked as a postdoc in ISIR, UPMC, France for 2 years. Then, he joined to LPN-CNRS as CNRS tenured researcher. His research interest covers microfluidics, lab-on-a-chip, in-situ SEM characterization, nanomanipulation.

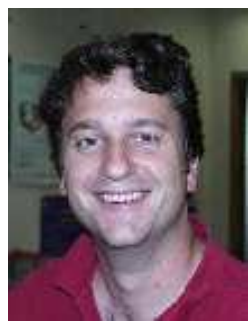

Nicolas Andreff received the Engineer degree ( M.S. degree, 1994) in Computer Sciences and Applied Mathematics from ENSEEIHT, Toulouse, France and the Ph.D. (1999) in Computer Graphics, Computer Vision and Robotics from INPG, Grenoble, France. He is currently Professor at the AS2M department of Institut FEMTO-ST, Université de Franche-Comté, Besançon, France. He created the Biomedical Micro-/Nano-Robotics group at AS2M department in 2012. His research interests range from vision-based control and parallel kinematics to intracorporeal microrobotics.

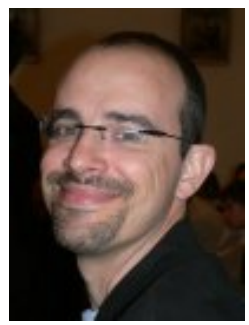

Stéphane Régnier received the Ph.D. degree in Mechanics and Robotics from the University of Pierre \& Marie Curie, Paris, France in 1996. He is currently Professor at the Institute of Intelligent Systems and Robotics (ISIR), University of Pierre $\&$ Marie Curie, Paris, France. He has been head of the ISIR micromanipulation team since 2001. His research interests are focused on micro and nano manipulation, teleoperation and haptic feedback at the nanoscale, micromechatronics and biological cell characterization. 